\title{
Immune Responseto Influenza Vaccination in Older Adults: Interactions with Type 2 Diabetes
}

\author{
Haleh Rokni, Nisha Nigil Haroon and Janet E McElhaney*
}

\author{
Health Sciences North Research Institute, Sudbury, Canada
}

Type 2 diabetes mellitus (T2DM) is one of the most common disorders among adults over age 65. Both humoral and cellular immune responses are negatively affected by diabetes [1]. Therefore infections such as influenza occur commonly with serious consequences in older individuals with T2DM. On the other hand aging is associated with changes in the immune system which result in disorders like T2DM and cardiovascular diseases [2]. Influenza is one of the most important causes of morbidity and mortality in both diabetic and non-diabetic older population and is associated with serious complications affecting cerebrovascular, cardiac and respiratory systems. The World Health Organization recommends the use of Influenza vaccine in people with diabetes and adults aged 65 years or older [3].

Several studies have shown that Influenza vaccination in older adults is associated with reduced hospital admissions [4]. However it is widely perceived that the benefits of influenza vaccination are less in patients with diabetes due to a dysregulation of the immune system. A study done by Looijmans and colleagues showed that vaccination was definitely effective in diabetic subjects [5]. Results of this nested case control study showed that immunisation against Influenza could significantly decrease hospitalisation in elderly diabetic patients. Cornelius Remschmidt and colleagues did a systemic review and showed that there is huge confounding in all studies done for evaluation of flu vaccine efficacy [6]. They suggest that randomised controlled trials or quasi-experimental studies with lab confirmed influenza diagnosis must be done. There are several studies that measured antibody responses to Influenza vaccination in both diabetic and healthy subjects and all of them showed no difference in efficacy based on serologic responses between two groups. Also, there was no difference in post vaccination antibody titers between healthy and diabetic subjects [7]. The study population included patients with Type 1 and Type 2 diabetes mellitus. Glycemic control is the main determinant of antibody response in those with diabetes whereas high-density lipoprotein - cholesterol (HDL-c) and white blood cell count predicts the antibody response in non-diabetic healthy subjects [8]. It is important to note that no studies have specifically compared antibody responses in subjects with poor glycemic status to those with good glycemic status. Cell-mediated immune responses are another important defense mechanism against influenza; $\mathrm{CD} 8^{+} \mathrm{T}$ cells are cytolytic $\mathrm{T}$ lymphocytes that are required for clearing virus from infected lung tissue [9]. The cytolytic mediators, granzyme $\mathrm{B}(\mathrm{GrB})$ and perforin, are released from $\mathrm{CD}^{+} \mathrm{T}$ cells and through a process mediated by insertion of perforin into the membrane of the infected host cell adjacent to the immunologic synapse, $\mathrm{GrB}$ gains access to the cytoplasm and triggers apoptotic cell death. We have found that persistent cytomegalovirus (CMV) infection in older adults results in the accumulation of late or terminally-differentiated $\mathrm{CD}^{+} \mathrm{T}$ cells containing GrB [10], which when released in the absence of perforin accumulates in extracellular space and causes tissue damage. This negatively impacts the T-cell response to influenza vaccination and overshadows any effect of T2DM in older adults with good glycemic control, in whom no effect of T2DM on T-cell response was found. However the study has very small sample size and as a result the lack of power is a concern. In addition, the role of CMV in the impaired antibody response to influenza vaccination in older adults is also independent of T2DM status [8]. Both studies are done in Canada and it is suggested that large studies be done in US.

Thus, older adults with T2DM who have good glycemic control are predicted to experience the same benefit from influenza vaccination as their healthy older counterparts. However, further studies are needed to determine how this benefit may be altered in those older diabetics with poor glycemic control. In those older diabetics with poor glycemic control and those who have progressed to insulin-requiring T2DM, lower functional reserve and increased susceptibility to the complications of influenza would be expected although more difficult to detect at a population level. The associated loss of influenza vaccine effectiveness and increased risk for complications of influenza leading to hospitalization, may frequently lead to irreversible functional decline [11]. This loss of functional reserve measured as the level of frailty is an important predictor of the response to influenza vaccination in both healthy and diabetic older adults [8]. Thus, future studies evaluating both antibody and cellmediated immune responses to influenza vaccination in the larger diabetic patient population are needed to understand the impact of $\mathrm{T} 2 \mathrm{DM}$ on influenza vaccine effectiveness given its prevalence in the over 65 population.

*Corresponding author: Dr. Janet E McElhaney, Health Sciences North Research Institute, 41 Ramsey Lake Road, Sudbury, ON P3E 5J1, Canada, Tel: 705-523-7100/2725, Fax: 705-523-7079, E-mail: jmcelhaney@hsnri.ca

Received: November 28, 2016: Accepted: January 18, 2017: Published online: January 21, 2017

Citation: Rokni H, Haroon NN, McElhaney JE (2017) Immune Response to Influenza Vaccination in Older Adults: Interactions with Type 2 Diabetes. Clin Diabetes Res 1(1):6-7

Copyright: (c) 2017 Rokni H, et al. This is an open-access article distributed under the terms of the Creative Commons Attribution License, which permits unrestricted use, distribution, and reproduction in any medium, provided the original author and source are credited. 
Citation: Rokni H, Haroon NN, McElhaney JE (2017) Immune Response to Influenza Vaccination in Older Adults: Interactions with Type 2 Diabetes. Clin Diabetes Res 1(1):6-7

\section{References}

1. Muller LM, Gorter KJ, Hak E, et al. (2005) Increased risk of common infections in patients with type 1 and type 2 diabetes mellitus. Clin Infect Dis 41: 281288

2. Pawelec G, Goldeck D, Derhovanessian E (2014) Inflammation, ageing and chronic disease. Curr Opin Immunol 29: 23-28.

3. Doherty M, Schmidt-Ott R, Santos JI, et al. (2016) Vaccination of special populations: Protecting the vulnerable. Vaccine 34: 6681-6690.

4. Colquhoun AJ, Nicholson KG, Botha JL, et al. (1997) Effectiveness of influenza vaccine in reducing hospital admissions in people with diabetes. Epidemiol Infect 119: 335-341.

5. Looijmans-Van den Akker I, Verheij TJ, Buskens E, et al. (2006) Clinical effectiveness of first and repeat influenza vaccination in adult and elderly diabetic patients. Diabetes care 29: 1771-1776.
6. Remschmidt C, Harder T, Wichmann O, et al. (2016) Effectiveness, immunogenicity and safety of 23 -valent pneumococcal polysaccharide vaccine revaccinations in the elderly: a systematic review. BMC Infect Dis 16: 711.

7. Pozzilli P, Gale EA, Visalli N, et al. (1986) The immune response to influenza vaccination in diabetic patients. Diabetologia 29: 850-854.

8. McElhaney JE, Garneau H, Camous X, et al. (2015) Predictors of the antibody response to influenza vaccination in older adults with type 2 diabetes. BMJ Open Diabetes Res Care 3: e000140.

9. Srikiatkhachorn A, Braciale TJ (1997) Virus-specific CD8+ T lymphocytes downregulate $\mathrm{T}$ helper cell type 2 cytokine secretion and pulmonary eosinophilia during experimental murine respiratory syncytial virus infection. J Exp Med 186: 421-432.

10. McElhaney JE (2005) The unmet need in the elderly: designing new influenza vaccines for older adults. Vaccine 23: S10-S25

11. Thompson WW, Shay DK, Weintraub E, et al. (2004) Influenza-associated hospitalizations in the United States. JAMA 292: 1333-1340. 